\title{
Evaluación del sistema de potabilización de agua constituido por filtros de bioarena y dióxido de titanio ${ }^{\dagger}$
}

\author{
Gema Rivas, Eddysson Flores, Javier Ramírez* \\ Facultad de Ingeniería Química, Universidad Nacional de Ingeniería (UNI) \\ Avenida Universitaria, PO Box 5595, Managua, Nicaragua \\ (recibido/received: 05-Aug-2014; aceptado/accepted: 14-Nov-2014)
}

\begin{abstract}
RESUMEN
Un sistema de tratamiento (ST) de operación por lote conformado por un filtro de bioarena (FBA) y otro de medio adsorbente de dióxido de titanio (FMADT) para potabilizar agua fue evaluado durante 121 días, a través del desarrollo de la biocapa, monitoreo del ST, determinación de la calidad del agua cruda y filtrada, las eficiencias de remoción del FBA y FMADT, y monitoreo del arsénico en el efluente del ST. El desarrollo de la biocapa en la superficie de la arena de filtración del FBA requirió 31 días y éste funcionó estable en la remoción de turbiedad, cuando en el agua cruda cargada varió entre $<1$ hasta 31 NTU y disminuyendo el $\mathrm{pH}$ entre 7.6-8.3. La calidad del agua cruda de pozo "La Corona" sugirió que solamente el manganeso $(1.9 \mathrm{mg} / \mathrm{L} \mathrm{Mn})$ no cumplió con la NTON 05 007-98, mientras que en el agua filtrada por el ST con la conductividad $(502 \mu \mathrm{S} / \mathrm{cm})$ y la dureza cálcica $\left(53.91 \mathrm{mg} / \mathrm{L} \mathrm{CaCO}_{3}\right)$ los que exceden la normativa CAPRE. Las eficiencias de remoción más destacadas del FBA son 99.9, 98.46, 91.76 y $62.16 \%$ correspondientes a E. coli, turbiedad, hierro total y el amonio, respectivamente. Mientras, las del FMADT son 30\% para manganeso y 99.9\% para arsénico, removiéndolo de 40-60 $\mu \mathrm{g} / \mathrm{L}$ As del agua cruda hasta $<10 \mu$ $\mathrm{g} / \mathrm{L}$ As.
\end{abstract}

Palabras clave: Agua subterránea; Arsénico; Filtración; Adsorción

\begin{abstract}
A batch treatment system (TS) consisting of a biosand filter (BSF) and a filter system packed with titanium dioxide (FSTD) for purifying drinking water was evaluated during 121 days, through the development of the biofilm, TS monitoring, determining the quality of the raw and filtered water, removal efficiencies of BSF and FSTD, and monitoring of arsenic within the effluent TS. The development of the biofilm on the sand surface of the BSF required 31 days and it worked stable in removing turbidity when the raw water varied $<1$ up to $31 \mathrm{NTU}$ and decreasing the $\mathrm{pH}$ between 7.6-8.3. The quality of the raw water of "La Corona" suggested that only manganese $(1.9 \mathrm{mg} / \mathrm{L} \mathrm{Mn})$ did not fulfill with the NTON $05007-98$, while only the conductivity $(502 \mu \mathrm{S} / \mathrm{cm})$ and the calcium hardness $(53.91 \mathrm{mg} / \mathrm{L} \mathrm{CaCO})$ exceeded CAPRE regulations for the filtered water. The top removal efficiencies of the BSF were $99.9 \% ; 98.46 \% ; 91.76 \%$ and $62.16 \%$ corresponding to E. coli, turbidity, total iron and ammonium, respectively. Meanwhile, the top removal efficiencies of the FSTD were $30 \%$ for manganese and $99.9 \%$ for arsenic, removing $40-60 \mu \mathrm{g} / \mathrm{L}$ As of the raw water up to $<10 \mu \mathrm{g} / \mathrm{L}$ As.
\end{abstract}

Keywords: Groundwater; Arsenic; Filtration; Absorption

†Dedicado al Prof. Dr.-Ing. Joaquín Martínez (KTH/Suecia) con motivo de su 65 cumpleaños.

\footnotetext{
*Autor para la correspondencia. Tel.: +505 22781463.

Correo electrónico: jramirez@uni.edu.ni (J. Ramírez).
} 


\section{Introducción}

La problemática en Nicaragua acerca del deterioro de la calidad del agua que se abastece a los consumidores fue descrita por Giraldo (2004) como un fenómeno común en los países en desarrollo, debido a la deficiente utilización de los recursos hídricos, las descargas de aguas residuales sin tratamiento, las limitaciones de infraestructura para el tratamiento de agua para el consumo, los efectos antropogénicos de las actividades humanas y desastres naturales, cuyos resultados se manifiestan en una serie de enfermedades de origen hídrico causadas por microorganismos patógenos que son nocivos para la salud de las poblaciones.

También los mecanismos geológicos representan uno de los fenómenos más peligrosos, porque pueden movilizar sustancias químicas muy tóxicas que existen naturalmente en los sedimentos y capas de los acuíferos que contiene al agua subterránea, como es el caso del arsénico inorgánico, un contaminante cancerígeno para los seres humanos que afecta a muchas regiones del mundo incluyendo Nicaragua, y cuya presencia en el agua subterránea puede exceder el nivel de concentración que provoca intoxicación crónica a las personas (Bang et al., 2005).

Dadas las condiciones que anteceden, las familias de las comunidades rurales que viven sin acceso a agua segura y sin servicios de saneamiento, son las que se encuentran en permanente riesgo de contraer enfermedades hídricas e intoxicarse con sustancias químicas como el arsénico (Aurazo, 2004; OPS/OMS, 2001). En el caso específico de la comunidad "La Corona", la ONG "El Porvenir" de DenverColorado determinó en el pozo comunitario un rango de concentración de 46-56 $\mu \mathrm{g} / \mathrm{L}$ As. Como consecuencia, la ONG "El Porvenir" en conjunto con la Organización Aqua Clara International de la Universidad Estatal de Michigan decidieron proceder con la instalación de un sistema de tratamiento (ST) constituido por un filtro de bioarena (FBA) y uno de medio adsorbente de dióxido de titanio (FMADT) en 16 viviendas de la comunidad, durante los meses de Octubre y Noviembre del año 2012.
El FBA que instaló la Organización Aqua Clara Nicaragua está constituido por: la capa de arena fina de filtración, la capa de arena gruesa para separación y la capa de piedrín para drenaje. Su principal función es la de remover los sólidos suspendidos y los microorganismos patógenos, mediante un entrampamiento mecánico, depredación, adsorción y muerte natural en la biocapa y la arena de filtración. Según CAWST (2012), la biocapa es el componente clave del filtro debido a que aumenta la eficiencia del tratamiento removiendo hasta un $99 \%$ de los patógenos. El FMADT está conformado por distintas capas de piedrín, arena fina, grava y carbón activado impregnado con plata pero la principal es la del dióxido de titanio. Éste adsorbe el arsénico inorgánico por complejación superficial en un rango de $\mathrm{pH}$ 6.5-8.5 debido a las fuerzas de naturaleza covalentes con efectos irreversibles (Bang et al., 2005; Guan et al., 2012; Valencia, 2007).

Es en esta prospectiva en la que se llevó a cabo la instalación y evaluación del ST a escala real para el agua de pozo de la comunidad "La Corona"; conformado por un FBA y un FMADT conectados en serie bajo operación por lote. La evaluación tecnológica del ST contribuiría a identificar si este sistema favorece obtener agua potable segura para los consumidores.

\section{Material y Métodos}

\subsection{Ubicación del pozo comunitario La Corona}

La comunidad "La Corona" se encuentra a 13 km del municipio de Camoapa del departamento de Boaco. El pozo comunitario está situado a una altura de $486 \mathrm{~m}$ sobre el nivel del mar y en las coordenadas: latitud $12^{\circ} 26^{\prime} 46.00^{\prime \prime} \mathrm{N}$ y longitud $85^{\circ} 30^{\prime} 35.56 " \mathrm{O}$. Por tanto, esta fuente sirvió como el punto de recolección de agua cruda utilizada en el ST evaluado.

\subsection{Desarrollo de la capa biológica en el filtro de bioarena}

Para lograr un buen desarrollo de la capa biológica en el FBA se utilizaron tres tipos de agua cruda: 1) agua cruda de pozo UNI-RUSB (AC-1) recolectada en un segmento de tubería 
(bypass) comprendido entre la salida de la bomba mecánica del pozo y el tanque de almacenamiento de este mismo recinto universitario; 2) agua cruda preparada a partir de una mezcla que contenía $10 \mathrm{~L}$ de agua de pozo UNI-RUSB y 2 L de agua del Lago Xolotlán (AC-2). El agua del lago Xolotlán se recolectó en el Puerto Salvador Allende de la ciudad de Managua-Nicaragua; 3) agua cruda preparada a partir de una mezcla que contenía $10 \mathrm{~L}$ de agua de pozo "La Corona" y 2 L de agua del Lago Xolotlán (AC-4). Para posteriores designaciones se le ha nombrado al agua cruda de pozo "La Corona" como AC-3.

El proceso de desarrollo de la capa biológica se realizó durante 31 días, cargando el FBA con dos lotes diarios de agua cruda de $12 \mathrm{~L}$ cada uno.

Paralelo a este procedimiento, se midió la turbiedad de entrada y salida del FBA porque se utilizó como parámetro de seguimiento al desarrollo de la capa biológica, debido a que se ha demostrado que el incremento en la turbiedad del agua tratada aumenta el riesgo de transportar partículas de un tamaño semejante al de los quistes de protozoarios (como la Giardia y el Cryptosporidium) e incluso puede llegar a enmascarar a virus y bacterias (Aurazo, 2004).

\subsection{Monitoreo del sistema de tratamiento}

El monitoreo del ST tuvo una duración de 121 días, consistiendo en el monitoreo del FBA y el FMADT acoplados, lo cual se logró cargando el ST con AC-1, AC-3 y AC-2.

En el transcurso del monitoreo se midieron parámetros como turbiedad y $\mathrm{pH}$. En la última etapa del monitoreo, la turbiedad y el $\mathrm{pH}$ medido se utilizaron para establecer el comportamiento de los filtros, una vez que se encontraban conectados y éstos parámetros se determinaron con el turbidímetro portátil Hach modelo 2100P y el pH-metro de bolsillo MARTINI ${ }^{\circledR}$ modelo $\mathrm{pH} 56$, respectivamente.

En la Tabla 1 se muestran los parámetros medidos en el ST en los días correspondientes del monitoreo.

\subsection{Puntos de recolección de muestras en el sistema de tratamiento}

En la Fig. 1 se muestra el ST (FBA + FMADT), evaluado con tres puntos de recolección de muestras (PRM). La importancia que radicó en los tres PRM indicados en la Fig. 1, se puntualizan de la siguiente forma:

- Punto de recolección de muestra 1 (PRM-1) a la entrada del ST: se ejecutaron tres muestreos correspondientes a una frecuencia de muestreo rigurosa durante las estaciones climáticas comprendidas entre el periodo de Noviembre 2012 a Enero 2013 para examinar el agua cruda de pozo "La Corona".

- Punto de recolección de muestra 2 (PRM-2) en el intermedio del ST: se realizaron dos muestreos, cumpliendo así con la frecuencia de muestreo estándar para analizar el agua filtrada por el FBA.

- Punto de recolección de muestra 3 (PRM-3) a la salida del ST: se efectuaron dos muestreos e igualmente se aplicó la frecuencia de muestreo estándar para determinar la calidad del agua filtrada por el FMADT.

2.5. Métodos de análisis aplicados en la determinación de la calidad del agua en el PRM-1, PRM-2 y PRM-3

Se analizaron 18 parámetros fisicoquímicos y 2 microbiológicos en cada uno de los PRM, los cuales fueron: turbiedad, $\mathrm{pH}$, coliformes totales y termotolerantes, temperatura, conductividad eléctrica, alcalinidad total, dureza total, calcio/dureza cálcica, magnesio y/o dureza magnésica, sólidos totales disueltos (STD), cloruros, sulfato, manganeso, hierro total, color verdadero, nitrito, nitrato, amonio y arsénico. Cuando se analizaron en PRM-1 se contrastaron con la NTON 05 007-98 y en PRM-3 con las normas CAPRE.

Los resultados de los diferentes ensayos fisicoquímicos y microbiológicos fueron obtenidos por la utilización de procedimientos analíticos normalizados, cuyos fundamentos y descripción están detallados en: 
Tabla 1 Monitoreo del ST de acuerdo a parámetros medidos.

\begin{tabular}{|c|c|c|c|c|c|c|c|}
\hline \multirow{3}{*}{$\begin{array}{l}\text { Parámetros } \\
\text { medidos }\end{array}$} & \multirow{3}{*}{ Unidades } & \multicolumn{6}{|c|}{ Monitoreado } \\
\hline & & \multicolumn{2}{|c|}{$\begin{array}{c}\text { A la entrada y salida } \\
\text { del FBA }\end{array}$} & \multicolumn{2}{|c|}{$\begin{array}{c}\text { En el PRM-1 y PRM-3 } \\
\text { del ST }\end{array}$} & \multicolumn{2}{|c|}{$\begin{array}{c}\text { En el PRM-1, PRM-2 y } \\
\text { PRM-3 del ST }\end{array}$} \\
\hline & & $\begin{array}{c}\text { Desde el } \\
\text { día }\end{array}$ & $\begin{array}{l}\text { Hasta el } \\
\text { día }\end{array}$ & $\begin{array}{l}\text { Desde el } \\
\text { día }\end{array}$ & $\begin{array}{l}\text { Hasta el } \\
\text { día }\end{array}$ & $\begin{array}{l}\text { Desde el } \\
\text { día }\end{array}$ & $\begin{array}{l}\text { Hasta el } \\
\text { día }\end{array}$ \\
\hline Turbiedad & NTU & 1 & 56 & 57 & $\begin{array}{c}89 \\
\left(1^{\text {er }} \text { lote }\right)\end{array}$ & $\begin{array}{c}89 \\
\left(2^{\text {do }} \text { lote }\right)\end{array}$ & 121 \\
\hline $\mathrm{pH}$ & $\begin{array}{l}\text { Unidades } \\
\text { estándar }\end{array}$ & - & - & 84 & 89 & 89 & 121 \\
\hline
\end{tabular}

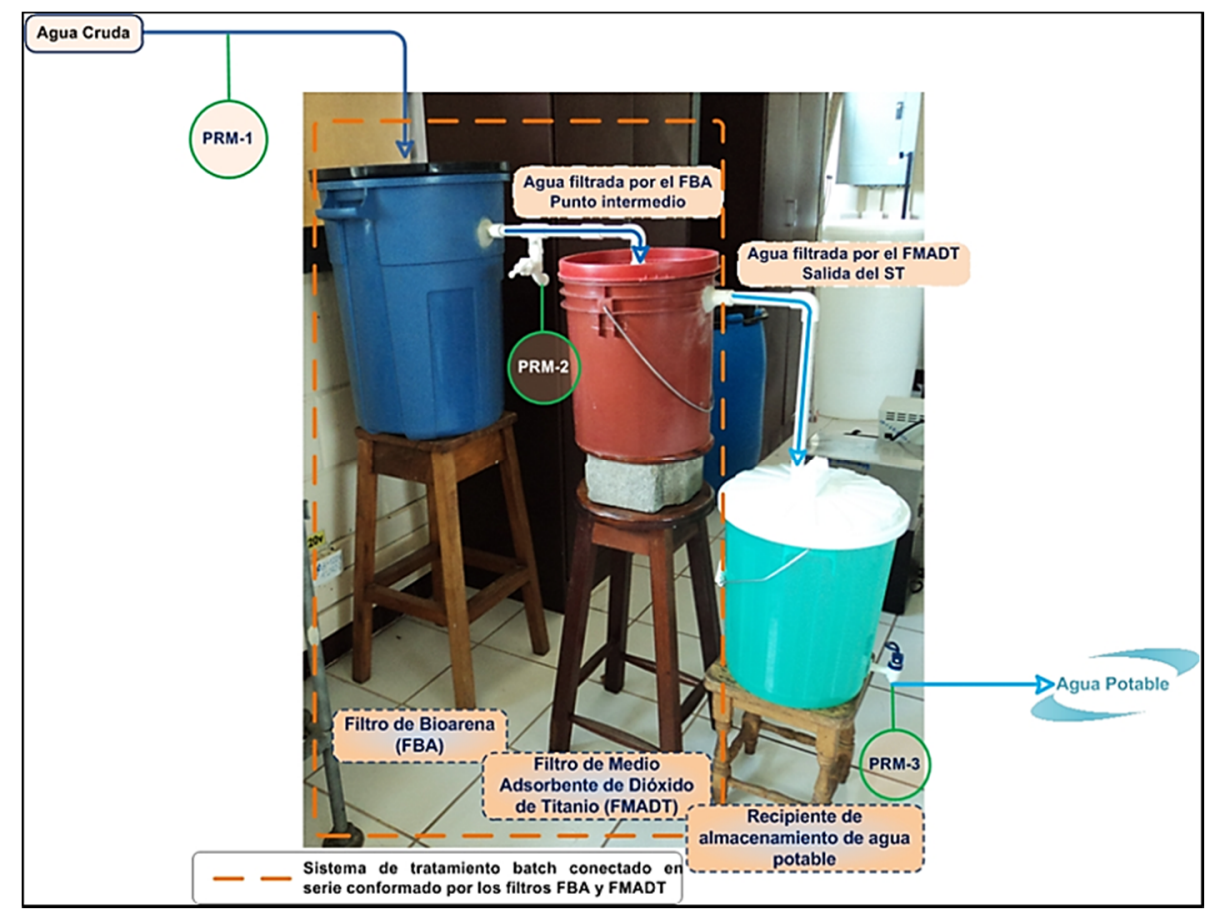

Fig. 1 Esquema del ST evaluado en el LIP con sus dos unidades de filtración conectadas en serie y sus respectivos PRM.

Standard Methods for the Examination of Water and Wastewater (Rice et al., 2005), DR5000 UV-Vis Spectrophotometer: Procedures manual (Hach Company, 2005), Placas 3M Petrifilm ${ }^{\mathrm{TM}}$ para el recuento de $E$. coli/coliformes y método colorimétrico de Gutzeit para arsénico (Wagtech, 2012).

Cabe destacar, que en la medición de las concentraciones de los parámetros fisicoquímicos determinados por los métodos de Hach Company (2005), se utilizaron reactivos marca Hach. En el resto de parámetros fisicoquímicos medidos, se emplearon reactivos químicos grado reactivo marca Fisher Scientific, Sigma Aldrich y Merck con cristalería de volumen preciso serie A y B. Además, todas las soluciones utilizadas fueron preparadas con agua destilada y fueron estandarizadas de acuerdo a los procedimientos contenidos en Rice et al. (2005).

2.6. Eficiencia de remoción para el ST, FBA y FMADT

Por medio de la Ecuación (1) se calcularon las eficiencias de remoción que evaluaron la eficacia de los procesos de filtración y adsorción 
que se produjeron en el ST así como en cada una de las unidades que lo conforman, cuyas acciones removieron los constituyentes del AC-3 cargado.

$$
E f_{R}=\frac{M P_{p e}-M P_{p s}}{M P_{p e}}
$$

donde $E f_{R}$ es la eficiencia de remoción, $M P_{p e}$ es la magnitud del parámetro en el punto de entrada del ST-unidad de filtración, $M P_{p s}$ es la magnitud del parámetro en el punto de salida del STunidad de filtración.

2.7. Monitoreo de la concentración de arsénico inorgánico en el efluente del sistema de tratamiento

Para determinar la concentración de arsénico inorgánico a la salida del FMADT, se monitorearon entre los días 87 y 108 distintos lotes del AC-3 procesados por este filtro. Para ello se recolectaron 15 muestras de $150 \mathrm{~mL}$, cada una en frascos de muestra de plástico de $200 \mathrm{~mL}$, las cuales se resguardaron bajo refrigeración para su preservación y posterior análisis con el equipo Wagtech Arsenator ${ }^{\circledR}$ Digital Arsenic Test Kit System.

De las 15 muestras recolectadas, 6 de ellas correspondieron a los efluentes obtenidos de Lotes 1, y 9 de efluentes de Lote 2; esto con el propósito de analizar la influencia del tiempo de residencia en el FMADT $\left(\tau_{\mathrm{FMADT}}\right)$, porque éste es el que tiene la función en el ST de remover el arsénico inorgánico del agua. El $\tau_{\mathrm{FMADT}}$ se define como el tiempo comprendido entre la carga de un lote que provoque el desplazamiento del agua retenida en el FBA hacia el FMADT y la carga de un lote subsiguiente que provoque la salida del agua retenida en el FMADT. Las muestras fueron analizadas en el Departamento de Salud Ambiental del Centro Nacional de Diagnóstico y Referencia-Ministerio de Salud (CNDRMINSA) con el equipo mencionado.

\section{Resultados y Discusión}

\subsection{Desarrollo de la capa biológica en el filtro de bioarena}

Durante el periodo de tiempo comprendido entre el día 13 al 44, se logró el desarrollo de la capa biológica (biocapa o schmutzdecke) en los 1-2 $\mathrm{cm}$ de la superficie de la arena de filtración en el FBA, cabe señalar que durante los días 24 al 39 el FBA se cargó con AC-2. Este hecho que se refleja en la Fig. 2, ya que a partir del $2^{\text {do }}$ lote del día 39; el FBA comienza a estabilizarse con respecto a la remoción de la turbiedad en PRM2 , lo cual puede ser una evidencia que indica que el crecimiento de la capa biológica ha alcanzado un apto desarrollo.

Al centrar el análisis en la Fig. 2 se observa que durante el periodo de tiempo comprendido entre el $1^{\text {er }}$ lote del día 13 y el $1^{\text {er }}$ lote del día 24 en el que se cargaron los 21 lotes de $\mathrm{AC}-1$, el comportamiento de la turbiedad en el PRM-2 con respecto a la turbiedad del PRM-1 era inestable, debido a que en dos ocasiones (señaladas por las áreas entre curvas (2) y (4)) la turbiedad en el PRM-2 fue mayor a la turbiedad en el PRM-1, mientras que en las áreas entre curvas (1) y (3) ocurrió todo lo contrario. Este comportamiento entre la turbiedad en el PRM-1 y el PRM-2 puede explicarse fundamentalmente por el mecanismo de desprendimiento, la frecuencia de utilización del FBA y la razón flujo de sustrato. Con respecto a éste último, Arboleda (2000) y CAWST (2012) argumentan que al incrementar el flujo de sustrato que llega al FBA a través del agua cruda, aumenta la acumulación y crecimiento de biomasa entre los granos de la arena de filtración y en consecuencia proporciona las condiciones para remover en el proceso de filtración a microorganismos perjudiciales, turbiedad, hierro $\mathrm{y}$ en algunos casos el color, pero ocurre lo contrario cuando el flujo de sustrato que llega al FBA es bajo.

Los argumentos anteriores, dieron la pauta para cargar el FBA con agua cruda con mayor proporción de sustratos; siendo la utilizada para propósito del desarrollo de la capa biológica, el $\mathrm{AC}-2$, la cual fue una mezcla de $10 \mathrm{~L}$ de agua de pozo UNI-RUSB $+2 \mathrm{~L}$ del agua del lago Xolotlán y cargada entre el $2^{\text {do }}$ lote del día 24 y el $2^{\text {do }}$ lote del día 39. Además, en la Fig. 2 se aprecia que durante el periodo de tiempo total que se cargó el FBA con AC-2 se produjo otra 
inestabilidad, donde la turbiedad en el PRM-2 fue mayor a la del PRM-1. Este comportamiento tuvo lugar entre el $1^{\text {er }}$ lote del día 29 y el $1^{\text {er }}$ lote del día 31, especificado con el área entre curvas (5). A partir del día $1^{\mathrm{er}}$ lote del día 40 hasta el $2^{\mathrm{do}}$ lote del día 44 (área entre curvas (6) se empezó a cargar el FBA con el AC-4. Esta mezcla que consistió de 10 L de agua de pozo "La Corona" y 2 L de agua del lago Xolotlán. El área entre curvas (6) muestra el momento especifico de la estabilización de la remoción de la turbiedad por parte del FBA.

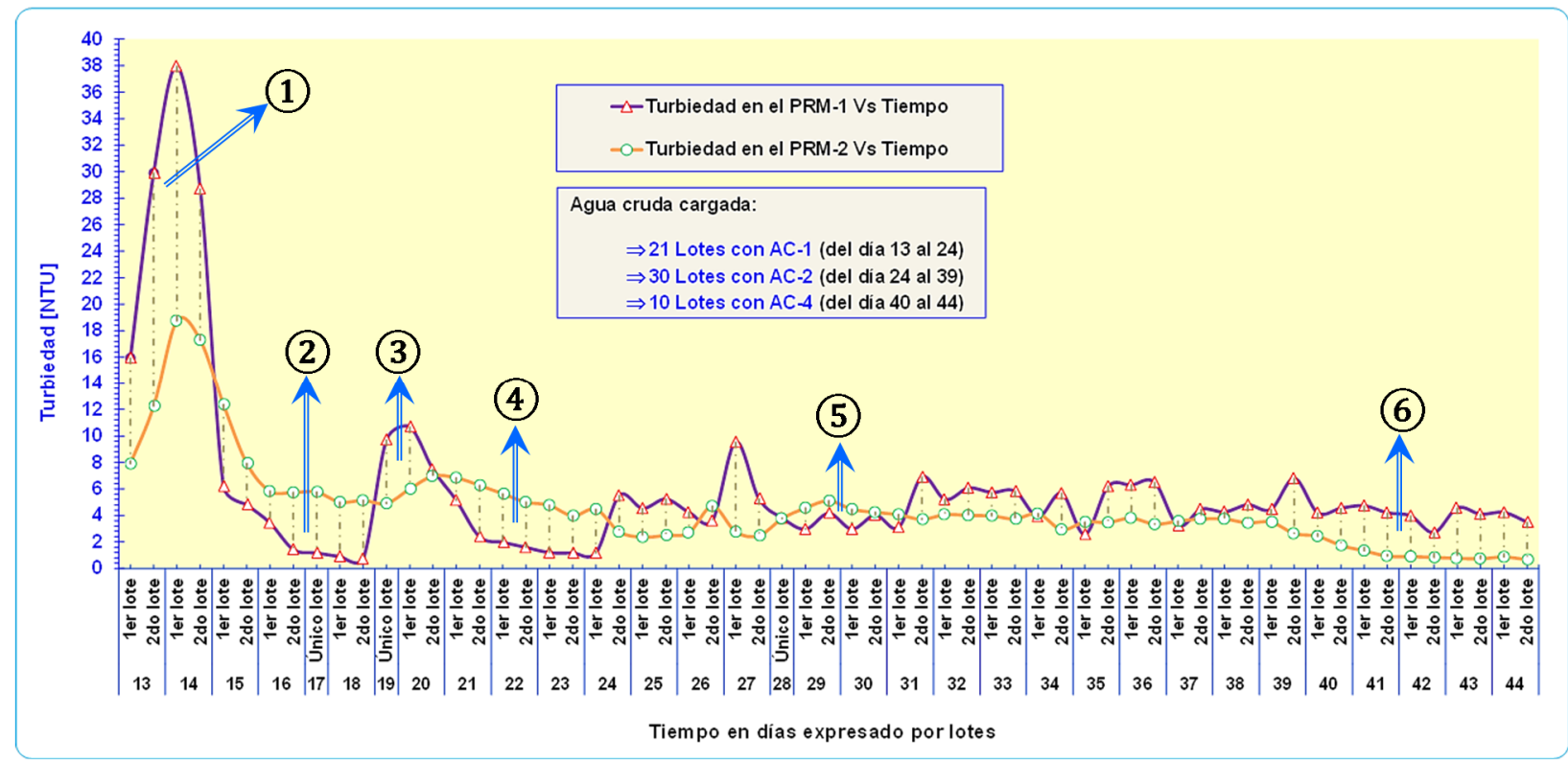

Fig. 2 Proceso de desarrollo de la capa biológica en el FBA descrito por el comportamiento de la turbiedad en el PRM-1 y PRM-2.

La explicación del mecanismo de desprendimiento es muy importante para esclarecer el por qué se producen el comportamiento donde la turbiedad en el PRM-2 es mayor que la del PRM-1.

Según Arboleda (2000), afirma que durante el proceso de filtración es normal que se produzcan frecuentes desprendimientos de partículas depositadas e incluso en forma de avalanchas de flóculos de partículas que se desprenden de los granos, debidos al esfuerzo cortante provocado por el agua que se carga al FBA, pero también plantean que cuando se bloquea una porción creciente del medio filtrante, el flujo se hace paso directo a través de cavidades no obstruidas por depósitos, donde la velocidad es demasiada alta para permitir su remoción en los granos circundantes a tal punto que atraviesan el medio filtrante, y salen en el efluente.

\subsection{Monitoreo del sistema de tratamiento}

En la Fig. 3 se describe la tendencia de la remoción de turbiedad en el FBA entre los días 90 al 121 conforme al comportamiento de la turbiedad en PRM-1 y PRM-2, donde en general se presenta cómo reacciona el FBA ante el brusco contraste de cargar el FBA con agua cruda con alta turbiedad y baja turbiedad. En el caso de la alta turbiedad cargada es connotada por el área entre curvas (1) y (3). El área entre curvas (1) se produjo entre el $1^{\text {er }}$ lote del día 90 y el $1^{\text {er }}$ lote del día 94, cuando el FBA se cargó con AC-3, cuyas turbiedades en PRM-1 variaron entre 6.80 NTU y 31 NTU, el FBA respondió con turbiedades en PRM-2 menores a 1 NTU, específicamente en un rango de respuesta entre 0.28 NTU a 0.59 NTU. El área entre curvas (3) estuvo constituida entre el $1^{\mathrm{er}}$ lote del día $110 \mathrm{y}$ el $2^{\text {do }}$ lote del día 121 para dar un total de 24 lotes cargados al FBA con AC-2, cuyas turbiedades en PRM-1 oscilaron entre 14.13 NTU y 23.33 NTU. Sin embargo, en esta ocasión el FBA presentó turbiedades en PRM-2 
en un rango de respuesta entre 0.27 NTU y 3.27 NTU, el cual se encuentra por debajo de los 5 NTU, que es el valor máximo admisible (VMA) establecido por las normas regionales para la calidad del agua potable CAPRE El caso de la baja turbiedad cargada al FBA es indicada por el área entre curvas (2), periodo para el cual se cargó con 29 lotes del AC-3 y fue comprendido entre el $2^{\text {do }}$ lote del día 94 y el $2^{\text {do }}$ lote del día 109 con turbiedades en el PRM-1 que fluctuaron entre 0.48 NTU y 1.51 NTU. El rango de respuesta por parte del FBA para estos 29 lotes cargados con baja turbiedad fue entre $0.23 \mathrm{NTU}$ a $0.75 \mathrm{NTU}$, indicado un rango muy por debajo del valor recomendado por CAPRE de 1 NTU para la turbiedad del agua potable.

Los resultados anteriores indican que el FBA funcionó de forma muy estable inclusive cuando se cargó agua cruda con turbiedades muy variables. Según Arboleda (2000) este grado de remoción tiene relación directa con el desarrollo de la capa biológica y con el tiempo que haya estado el FBA en servicio, de tal manera que al acumularse más sólidos suspendidos sobre la superficie de los granos de la arena de filtración los mecanismos de adherencia y cernido son más eficientes.

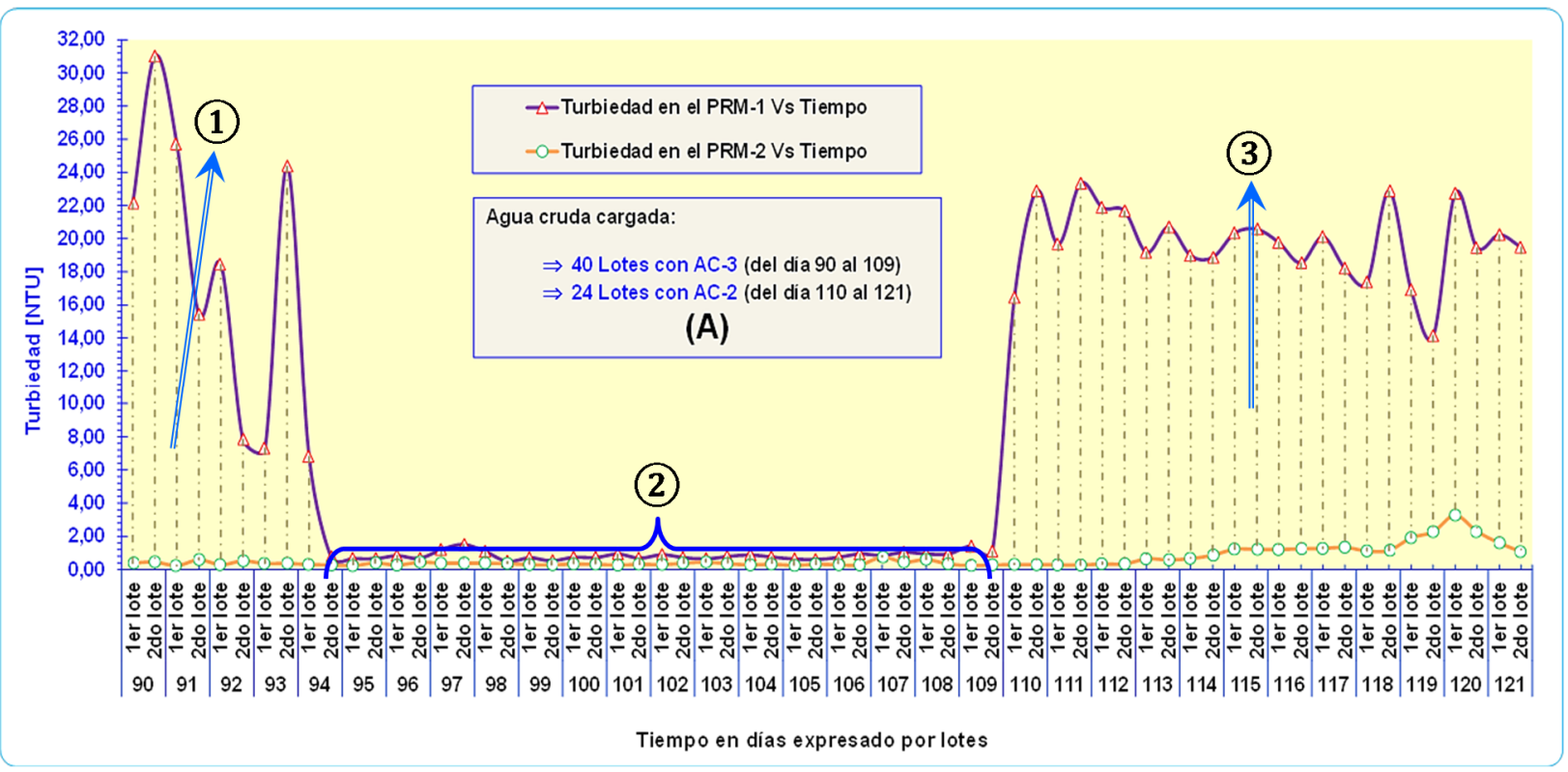

Fig. 3 Comportamiento de la turbiedad durante los días 90 al 121 en PRM-1 y PRM-2.

Los resultados del monitoreo del pH en PRM-1, PRM-2 y PRM-3 son resumidos esquemáticamente en la Fig. 4, donde se muestra cómo se comporta el pH en el ST y en sí en el FBA y el FMADT durante 31 días (del día 90 al 121) cuando se cargó con AC-2 y AC-3. En la Fig. 4 se describe como el FBA tenía la tendencia general de disminuir el $\mathrm{pH}$ de entrada del agua cruda a tratar, posterior a ser filtrada por su medio filtrante. Con base al total de los 62 lotes cargados (desde el $1^{\text {er }}$ lote del día 90 hasta el $2^{\text {do }}$ lote del día 121) y considerando los resultados de $\mathrm{pH}$ en el PRM-1 y PRM-2, se obtiene que el FBA: Incrementó los valores del
$\mathrm{pH}$ en el PRM-2 en el $12.90 \%$ de los lotes, disminuyó las magnitudes del $\mathrm{pH}$ en el PRM-2 en el $82.26 \%$ de los lotes y ambos resultados del pH en el PRM-1 y el PRM-2, se mantuvieron aproximadamente iguales en $4.84 \%$ de los lotes procesados por el FBA.

El rango de $\mathrm{pH}$ del agua cruda cargada (AC-2 y AC-3) al FBA desde el día 90 hasta el día 121 osciló entre 7.40 y 8.80 para lo cual una vez filtrada el agua por el FBA, producir un rango de respuesta en el PRM-2 para el $\mathrm{pH}$, comprendido entre 7.60 y 8.30 . Este rango de respuesta para el $\mathrm{pH}$ en el PRM-2 producido por el FBA se 
encuentra dentro del rango recomendado para el $\mathrm{pH}(6.5$ a 8.5) del agua potable establecido por CAPRE. Weiner (2008) explica por qué el FBA disminuye el $\mathrm{pH}$ de agua que filtra, lo cual se pude deber a la difusión del $\mathrm{CO}_{2}$ provocada por la actividad microbiana de la capa biológica.
Por otro lado, Chapman (1996) cita que los compuestos naturales tales como ácidos húmico y fúlvico controlan el $\mathrm{pH}$, por ende se puede deducir que la remoción de éstos compuestos naturales por parte de la capa biológica influye en la disminución del $\mathrm{pH}$.

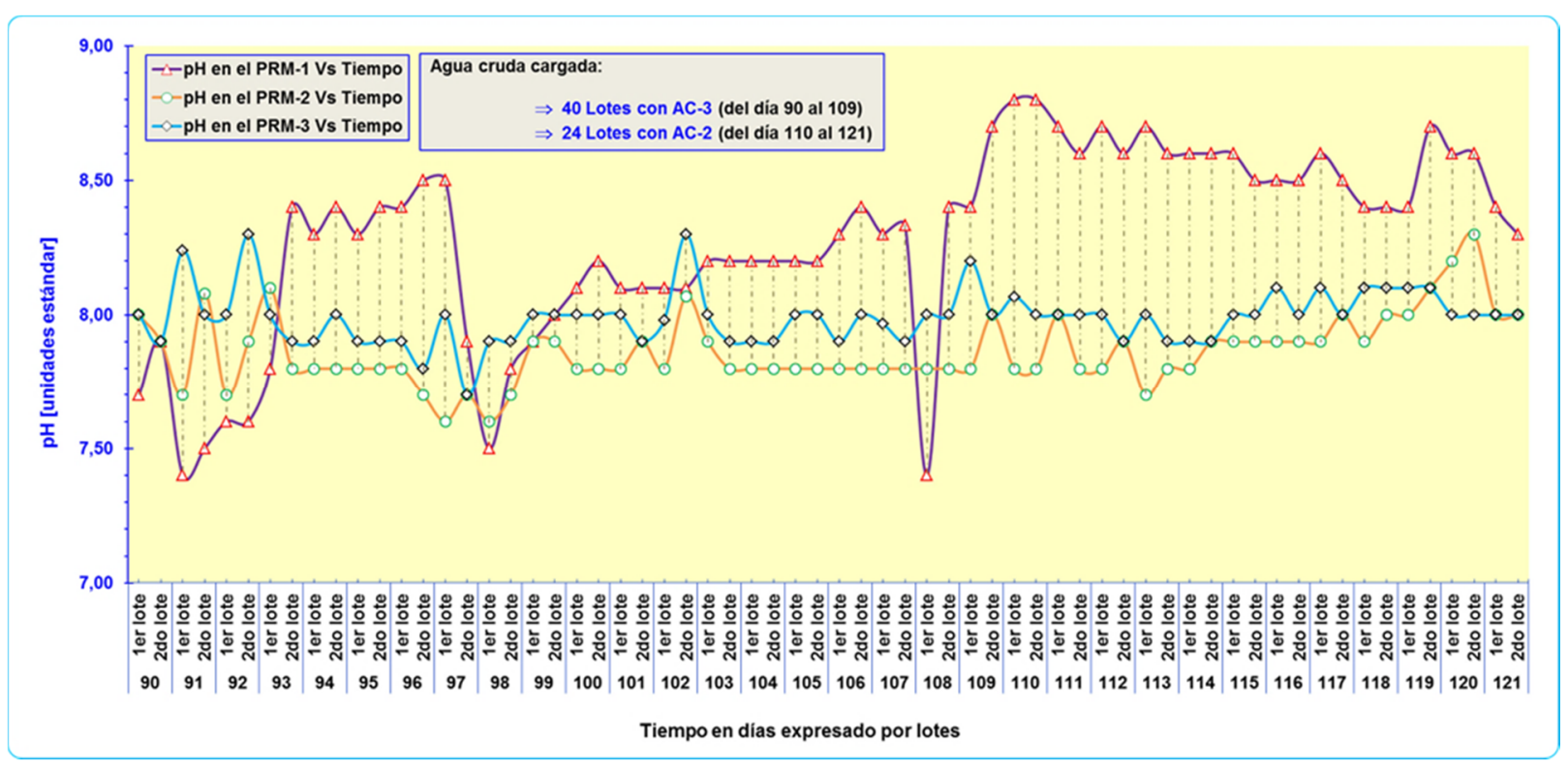

Fig. 4 Comportamiento del pH durante los días 90 al 121 en PRM-1, PRM-2 y PRM-3.

Cuando se compara el $\mathrm{pH}$ en PRM-2 con respecto al $\mathrm{pH}$ de $\mathrm{PRM}-3$, se aprecia la particularidad del FMADT para incrementar el pH del agua que éste procesa. Ante el rango de respuesta para el $\mathrm{pH}$ que produce el FBA en PRM-2 (ya mencionado), el FMADT responde con un rango de respuesta que varío entre 7.70 y 8.30. Bang et al. (2005) reportaron como parte de sus resultados que en los experimentos de adsorción de arsénico inorgánico utilizando $\mathrm{TiO}_{2}$ anatasita granular, se produjo una disminución leve del $\mathrm{pH}$ del agua tratada. Por lo tanto, es evidente que el comportamiento del $\mathrm{pH}$ descrito por el agua tratada por el FMADT no concuerda con el determinado por Bang et al. (2005).

No obstante, este comportamiento podría justificarse por la presencia de la capa de carbón activado en el FMADT, el cual posee cenizas no volátiles de óxidos metálicos, las cuales al entrar en contacto con el agua se disuelven en la misma convirtiéndose en hidróxidos, y por ende aumentado el $\mathrm{pH}$ a la salida del sistema purificador (Carbotecnia, 2010). Con los resultados del $\mathrm{pH}$ entre PRM-2 y PRM-3, se infiere que del total de los 62 lotes; el $17.74 \%$ presentaron magnitudes de $\mathrm{pH}$ muy cercanas, el $6.45 \%$ de los valores del $\mathrm{pH}$ en PRM-3 fueron menores a los del PRM-2 y el $75.81 \%$ de las magnitudes del pH en el PRM-3 fueron mayores a los del PRM-2.

El comportamiento del pH que ocurrió en el ST puede resumirse de la siguiente forma: $91.94 \%$ de los lotes procesados presentaron $\mathrm{pH}$ en PRM3 menores que las del PRM-1; 4.84\% de los lotes de agua cruda tratados por el ST mostraron resultados de $\mathrm{pH}$ en PRM-3 mayores a los del PRM-1 y $3.23 \%$ de total de los lotes indicaron que los valores del pH en el PRM-1 y el PRM-3 fueron aproximadamente iguales. Al analizar el comportamiento, se observa una tendencia generalizada en donde el ST fue capaz de disminuir el $\mathrm{pH}$ del agua cruda, y mantener el valor de $\mathrm{pH}$ en el agua filtrada a niveles dentro del rango recomendado por la norma CAPRE. 


\subsection{Calidad del agua cruda y filtrada en PRM- 1, PRM-2 y el PRM-3}

La Tabla 2 presenta los resultados en rangos de los análisis de los parámetros fisicoquímicos y microbiológicos para el afluente al FBA PRM1(AC-3) y los rangos de respuesta para el efluente del FBA (PRM-2) y efluente del ST (PRM-3), con sus respectivas unidades de medida y agrupados en niveles de vigilancia Conjuntamente, la tabla 2 muestra los límites o rangos máximos de la NTON 05 007-98 y los valores recomendados y admisibles de la Norma regional CAPRE para inferir acerca de la calidad del agua cruda y tratada por el ST respectivamente.

Los parámetros fisicoquímicos en el afluente al FBA que cumplen con el límite o rango máximo establecidos en la norma NTON 05 007-98 son: turbiedad, $\mathrm{pH}$, dureza total, STD, cloruros, sulfato, hierro, color verdadero, nitrito y nitrato, mientras que el manganeso no cumple, ya que el límite inferior (LI) del rango $(0.5 \mathrm{mg} / \mathrm{L} \mathrm{Mn})$ obtenido coincidió con el valor del límite máximo permitido. Los coliformes totales no se pueden contrastar con el rango máximo dado por la norma, debido a que es un promedio mensual y las pruebas microbiológicas fueron realizadas una sola vez en meses diferentes. El resto de los parámetros fisicoquímicos y microbiológicos como coliformes termotolerantes, temperatura (de cuarto), conductividad eléctrica, alcalinidad total, dureza cálcica, calcio, dureza magnésica, magnesio, amonio y arsénico no están contemplados en la norma.

Los parámetro fisicoquímicos y microbiológicos del efluente del ST (PRM-3) presentados en la Tabla 2, especifican que tanto la conductividad y la dureza magnésica/magnesio no cumplen con la norma regional CAPRE. La magnitud de la conductividad resultó igual a $502 \mu \mathrm{S} / \mathrm{cm}$, lo cual indica que dicho valor se mantuvo $102 \mu \mathrm{S} / \mathrm{cm}$ por encima del VR $(400 \mu \mathrm{S} / \mathrm{cm})$ según CAPRE. En el análisis del rango de respuesta de la dureza magnésica (45.72-53.9 $\left.\mathrm{mg} / \mathrm{L} \quad \mathrm{CaCO}_{3}\right)$, éste superó considerablemente al VR $(30 \mathrm{mg} / \mathrm{L}$ $\mathrm{CaCO}_{3}$ ) por CAPRE. Es importante señalar, que los VMA definen la calidad del agua para consumo humano como aquella que podrá consumirse con un mayor riesgo a la salud de los consumidores (Rojas, 2002).

Por otro lado, un análisis similar se puede aplicar para el magnesio, donde su LI se encontró $3.82 \mathrm{mg} / \mathrm{L} \mathrm{Mg}$ por encima del VR (7.29 $\mathrm{mg} / \mathrm{L} \mathrm{Mg}$ ) y así mismo, que su LS sobrepasaba en $0.95 \mathrm{mg} / \mathrm{L} \mathrm{Mg}$ al VMA de 12.15 $\mathrm{mg} / \mathrm{L} \mathrm{Mg}$. El resto de los parámetros se encuentran por debajo del valor máximo permitido y en el caso de: el STD, cloruros y sulfatos se encuentran por debajo del valor recomendado por la norma CAPRE.

Centralizando el análisis en el nivel básico de la Tabla 2, específicamente en los coliformes totales y termotolerantes, es claro que el agua cumple con lo establecido por la norma CAPRE, ya que estos resultaron ser menores a 1, lo cual se puede considerar negativo.

\subsection{Remoción de parámetros fisicoquímicos para el ST, FBA y FMADT}

La eficiencia de remoción de los parámetros fisicoquímicos en cada unidad de filtración y en general del ST para son presentadas en la Tabla 3. La remoción de la turbiedad alcanzada por el FBA y el ST fueron las más significativas dentro de todos los parámetros analizados. Sin embargo, el FBA demostró tener con un $98.46 \%$ la mejor remoción de la turbiedad, es decir, que la mayor proporción de turbiedad que entró al ST fue removida por el FBA e incluso éste porcentaje de remoción fue mayor al reportado por CAWST (2012). Cabe destacar, que la eficiencia de remoción igual a $98.46 \%$ significa que se obtuvo agua en PRM-2 con una turbiedad de $<1$ NTU. La remoción de hierro total alcanzada por el FBA fue de $91.67 \%$. Este dato está dentro del rango de remoción para el hierro total de $90-95 \%$ (ver Tabla 3) reportado por CAWST (2012) para el FBA. Para el caso de ion amonio se comprobó que el FBA remueve este en un $62.16 \%$ (de 0.37 a $0.14 \mathrm{mg} / \mathrm{L} \mathrm{NH} 4^{+}$). En ninguna de las referencias consultadas se encontró alguna eficiencia de remoción para el amonio en el FBA, por lo tanto, no se pudo comparar éstas eficiencias de remoción encontradas con algunas determinadas por diferentes autores en la literatura. 
Tabla 2 Calidad del agua de pozo "La Corona", y tratada por el FBA y el FMADT agrupada en niveles de vigilancia.

\begin{tabular}{|c|c|c|c|c|c|c|c|c|}
\hline \multirow{2}{*}{$\begin{array}{c}\text { Nivel de } \\
\text { vigilancia }\end{array}$} & \multirow{2}{*}{ Parámetro } & \multirow{2}{*}{ Unidades de medida } & \multirow{2}{*}{ Rango en PRM-1 } & \multirow{2}{*}{$\begin{array}{l}\text { Rango de respuesta } \\
\text { en el PRM-2 }\end{array}$} & \multirow{2}{*}{$\begin{array}{l}\text { Rango de } \\
\text { respuesta en el } \\
\text { PRM-3 }\end{array}$} & \multirow{2}{*}{$\begin{array}{c}\text { NTON } 05 \text { 007-98 } \\
\begin{array}{c}\text { Límite o rango } \\
\text { máximo }\end{array} \\
\end{array}$} & \multicolumn{2}{|c|}{ Norma regional CAPRE } \\
\hline & & & & & & & $\begin{array}{l}\text { Valor } \\
\text { recomendado }\end{array}$ & $\begin{array}{l}\text { Valor máximo } \\
\text { admisible }\end{array}$ \\
\hline \multirow{10}{*}{ 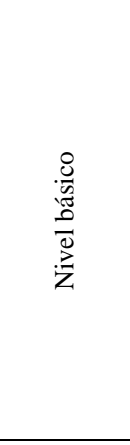 } & Turbiedad & NTU & $0.9-31.5$ & $0.38-0.59$ & $0.35-0.54$ & $<250$ & 1 & 5 \\
\hline & $\mathrm{pH}$ & Unidades estándar & $\begin{array}{c}7.48-7.79 \\
\left(\mathrm{a} \|=\mathrm{T}=20.7^{\circ} \mathrm{C}\right)\end{array}$ & $\begin{array}{c}8.07-8.08 \\
\left(25.4^{\circ} \mathrm{C}-28.9^{\circ} \mathrm{C}\right) \\
\end{array}$ & $\begin{array}{c}7.98-8.24 \\
\left(26^{\circ} \mathrm{C}-30^{\circ} \mathrm{C}\right) \\
\end{array}$ & $6.0-8.5$ & $6.5-8.5$ & - \\
\hline & Coliformes totales (FME) & $\mathrm{UFC} / 100 \mathrm{~mL}$ & $<1$ & $<1$ & $<1$ & + & - & - \\
\hline & Coliformes termotolerantes (FME) & $\mathrm{UFC} / 100 \mathrm{~mL}$ & $<1$ & $<1$ & $<1$ & $\dagger \dagger$ & Neg & Neg \\
\hline & Coliformes totales (PPEC) & $\mathrm{UFC} / 2 \mathrm{~mL}$ & $<1$ & $<1$ & $<1$ & - & - & - \\
\hline & Coliformes termotolerantes (PPEC) & $\mathrm{UFC} / 2 \mathrm{~mL}$ & $<1$ & $<1$ & $<1$ & - & - & - \\
\hline & Coliformes totales (FME + PPEC) & $\mathrm{UFC} / 100 \mathrm{~mL}$ & $<1$ & $<1$ & $<1$ & - & Neg & Neg \\
\hline & $\begin{array}{c}\text { Coliformes termotolerantes } \\
\text { (FME+ PPEC) }\end{array}$ & $\mathrm{UFC} / 100 \mathrm{~mL}$ & $<1$ & $<1$ & $<1$ & - & Neg & Neg \\
\hline & Temperatura (de cuarto) & ${ }^{\circ} \mathrm{C}$ & $21.05-23.6$ & $25-29$ & $25.5-30.5$ & - & $18-30$ & - \\
\hline & Conductividad eléctrica & $\mu \mathrm{S} / \mathrm{cm}$ & $\begin{array}{c}=503-505 \\
\left(20.8^{\circ} \mathrm{C}-26.3^{\circ} \mathrm{C}\right)\end{array}$ & $\begin{array}{c}502-503 \\
\left(21.9^{\circ} \mathrm{C}-25.9^{\circ} \mathrm{C}\right) \\
\end{array}$ & $\begin{array}{c}\ominus 502 \\
\left(21.9^{\circ} \mathrm{C}-22.7^{\circ} \mathrm{C}\right) \\
\end{array}$ & - & 400 & - \\
\hline \multirow{8}{*}{ 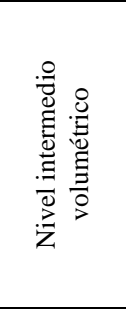 } & Dureza Total (EDTA) & $\mathrm{mg} / \mathrm{L} \mathrm{CaCO}_{3}$ & $137.33-149.08$ & $120.39-149.14$ & $136.05-140.51$ & 400 & 400 & - \\
\hline & Alcalinidad Total & $\mathrm{mg} / \mathrm{L} \mathrm{CaCO}_{3}$ & $\begin{array}{c}274.67-298.7 \\
\text { (a pH= 3.39-3.30) }\end{array}$ & $\begin{array}{c}292.48-293.48 \\
(\mathrm{a} \mathrm{pH}=3.48-3.55)\end{array}$ & $\begin{array}{c}288.62-293.83 \\
(\mathrm{a} \mathrm{pH}=3.45-3.57)\end{array}$ & - & - & - \\
\hline & Dureza cálcica & $\mathrm{mg} / \mathrm{L} \mathrm{CaCO}_{3}$ & $85.77-115.94$ & $91.92-107.34$ & $82.16-94.84$ & - & 100 & - \\
\hline & Calcio & $\mathrm{mg} / \mathrm{L} \mathrm{Ca}$ & $34.38-46.47$ & $36.84-43.02$ & $32.93-38.01$ & - & 40.08 & - \\
\hline & Dureza magnésica & $\mathrm{mg} / \mathrm{L} \mathrm{CaCO}_{3}$ & $21.39-51.99$ & $30.70-41.81$ & $45.72-53.91$ & - & 30 & 50 \\
\hline & Magnesio & $\mathrm{mg} / \mathrm{L} \mathrm{Mg}$ & $5.20-12.63$ & $7.46-10.16$ & $11.11-13.10$ & - & 7.29 & 12.15 \\
\hline & Sólidos totales disueltos (STD) & $\mathrm{mg} / \mathrm{L}$ STD & $332-348$ & $330-376$ & $361-364$ & 1.500 & - & 1000 \\
\hline & Cloruros & $\mathrm{mg} / \mathrm{L} \mathrm{Cl}^{-}$ & $10.50-11.81$ & $11.03-13.64$ & $11.02-11.94$ & 600 & 25 & 250 \\
\hline \multirow{8}{*}{ 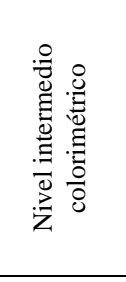 } & Sulfato & $\mathrm{mg} / \mathrm{L} \mathrm{SO}_{4}{ }^{2-}$ & $\mid=<2-2$ & $\ominus<2$ & $\ominus<2$ & 400 & 25 & 250 \\
\hline & Manganeso & $\mathrm{mg} / \mathrm{L} \mathrm{Mn}$ & $=0.5-1.9$ & $\ominus 0.5$ & $0.2-0.5$ & 0.5 & 0.10 & 0.50 \\
\hline & Hierro Total & $\mathrm{mg} / \mathrm{L} \mathrm{Fe}$ & $0.02-0.24$ & $\ominus<0.002$ & $\ominus<0.002$ & 3 & - & 0.30 \\
\hline & Color verdadero (a $455 \mathrm{~nm}$ ) & $\mathrm{mg} / \mathrm{L}$ Pt-Co & $1-4$ & $\ominus 2$ & $\ominus 1$ & $<150$ & 1 & 15 \\
\hline & $\begin{array}{c}\text { Nitrito } \\
\end{array}$ & $\mathrm{mg} / \mathrm{L} \mathrm{NO}_{2}^{-}-\mathrm{N}$ & $<0.002-2$ & $1-2$ & $<0.002-1$ & - & - & 1.0 \\
\hline & Nitrato & $\mathrm{mg} / \mathrm{L} \mathrm{NO}_{3}{ }^{-}-\mathrm{N}$ & $0.3-0.8$ & $0.7-0.9$ & $0.3-0.5$ & - & 5.65 & 11.29 \\
\hline & Nitritos + Nitratos $(\mathrm{N})$ & $\mathrm{mg} / \mathrm{L}\left(\mathrm{NO}_{2}^{-}-\mathrm{N}+\mathrm{NO}_{3}^{-}-\mathrm{N}\right)$ & $<0.302-2.8$ & - & - & 10.0 & - & - \\
\hline & Amonio & $\mathrm{mg} / \mathrm{L} \mathrm{NH}_{4}^{+}$ & $<0.02-0.37$ & $0.05-0.23$ & $0.04-0.18$ & - & 0.05 & 0.50 \\
\hline 离 & Arsénico & $\mu \mathrm{g} / \mathrm{L} \mathrm{As}$ & $42-46$ & - & $\ominus 0$ & - & - & 10 \\
\hline
\end{tabular}

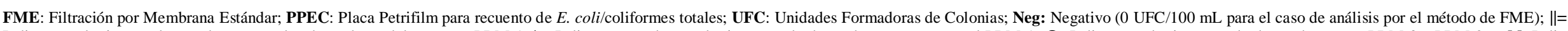

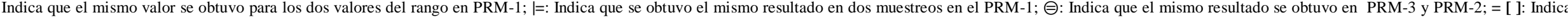

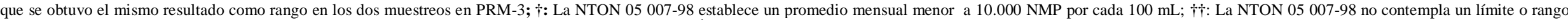
máximo para coliformes termotolerantes; nm: nanómetro; NTU: Nephelometric Turbidity Unit; EDTA: Ácido etilendiaminotetraacético. 
Tabla 3 Eficiencias de remoción para el FBA, el FMADT y el ST.

\begin{tabular}{|c|c|c|c|}
\hline Parámetro & $\begin{array}{c}\text { Eficiencia de } \\
\text { remoción } \\
\text { obtenida }\end{array}$ & $\begin{array}{l}\text { Eficiencia de remoción } \\
\text { documentada }\end{array}$ & Referencia \\
\hline \multicolumn{4}{|c|}{ Eficiencias de remoción para el FBA } \\
\hline Turbiedad & $98.46 \%$ & $95 \%$ & Buzunis (1995) \\
\hline Hierro Total & $91.67 \%$ & $90 \%-95 \%$ & Ngai et al. (2004) \\
\hline Amonio & $62.16 \%$ & - & - \\
\hline \multicolumn{4}{|c|}{ Eficiencias de remoción para el FMADT } \\
\hline Manganeso & $30 \%$ & $52.38 \%$ & Bang et al. (2005) \\
\hline Nitrito & $67 \%$ & - & - \\
\hline Nitrato & $50 \%$ & - & - \\
\hline Arsénico & $99.9 \%$ & $95.35 \%$ & Bang et al. (2005) \\
\hline \multicolumn{4}{|c|}{ Eficiencias de remoción para el ST } \\
\hline Turbiedad & $98.19 \%$ & \multirow{3}{*}{ - } & \multirow{3}{*}{ - } \\
\hline Amonio & $70.27 \%$ & & \\
\hline Arsénico & $99.99 \%$ & & \\
\hline
\end{tabular}

El Manganeso (Mn) que sale en el efluente del FBA fue removido hasta en $30 \%$ por el FMADT con lo que se logró reducir la concentración hasta una promedio de $0.35 \mathrm{mg} / \mathrm{L} \mathrm{Mn}$. Bang et al. (2005) reportó en los resultados de sus experimentos de campo una eficiencia de remoción de manganeso de hasta 52.38\%; utilizando columnas de $\mathrm{TiO}_{2}$ granular operadas por lotes.

La remoción del $\mathrm{NO}_{2}{ }^{-} \mathrm{-N}$ se redujo desde una concentración promedio de $1.5 \mathrm{mg} / \mathrm{L} \mathrm{NO}_{2}{ }^{-}-\mathrm{N}$ en PRM-2, hasta una concentración promedio de $0.50 \mathrm{mg} / \mathrm{L} \mathrm{NO}_{2}{ }^{-}-\mathrm{N}$ en PRM-3 con la cual se computó una eficiencia de remoción para el FMADT de $67 \%$. Esta remoción de nitrito posiblemente se debe a su oxidación en presencia de oxígeno a nitrato.

La concentración promedio de $0.8 \mathrm{mg} / \mathrm{L} \mathrm{NO}_{3}{ }^{-} \mathrm{N}$ del efluente del FBA se redujo en el FMADT hasta $0.4 \mathrm{mg} / \mathrm{L} \mathrm{NO}_{3}{ }^{-} \mathrm{N}$, lo que indicó que la eficiencia de remoción fue de 50\%. En la revisión de las referencias no se encontró información acerca de la eficiencia de remoción y/o mecanismos que tiene el $\mathrm{TiO}_{2}$ granular anatasita para remover nitrato. Además, no se puede atribuir la remoción del nitrato a un proceso de desnitrificación biológica, porque el FMADT no proporciona condiciones anóxicas.
El contenido de arsénico en AC-3 con concentración de $42 \mu \mathrm{g} / \mathrm{L}$ As fue removido hasta aproximadamente $0 \mu \mathrm{g} / \mathrm{L}$ As, en un rango de $\mathrm{pH}$ de 7.48-8.11 lo que generó una eficiencia de remoción de $99.90 \%$. De acuerdo a los experimentos de Bang et al. (2005), el arsénico fue removido en el agua subterránea de $43 \mu \mathrm{g} / \mathrm{L}$ As a $<2.0 \mu \mathrm{g} / \mathrm{L}$ As en un rango de $\mathrm{pH}$ de 7.917.90, obteniendo una eficiencia de remoción de $95.35 \%$, siendo la obtenida mayor a la reportado por Bang et al. (2005).

3.5. Monitoreo de la concentración de arsénico inorgánico en el efluente del sistema de tratamiento

Los resultados de las concentraciones de arsénico inorgánico a la salida del FMADT (o a la salida del ST) como función del tiempo de residencia $\left(\tau_{\text {FMADT }}\right)$ son presentados en la Tabla 4. Estos resultados demostraron las magnitudes de remoción alcanzadas por el $\mathrm{TiO}_{2}$ granular anatasita para varios lotes de AC-3 procesados por el FMADT pertenecientes entre los días 87 al 108. Los resultados de la Tabla 4, indican que la concentración de arsénico en PRM-3 es de 0 $\mu \mathrm{g} / \mathrm{L}$ para el $\tau_{\mathrm{FMADT}}$ más grande que fue de 40.67 h (día 108-lote 1), pero también demuestran que se obtiene el mismo resultado para el $\tau_{\mathrm{FMADT}}$ más pequeño de 6.57 h (día 101-lote 1 ). 
Por tanto, se podría considerar que con un tiempo de $6.57 \mathrm{~h}$ es suficiente para remover el arsénico presente en el agua. La forma en que el arsénico fue adsorbido por el $\mathrm{TiO}_{2}$ anatasita granular es complejación superficial debido a que el $\mathrm{pH}$ del AC-3 en PRM-2 durante los días del monitoreo de los lotes procesados en el
FMADT, siempre fueron mayores al PCC $=5.9$ y varió en un rango de 7.6-7.9 (Bang et al., 2005). Además, Guan et al. (2012) afirman que la complejación entre la superficie de $\mathrm{TiO}_{2}$ y diversas especies de arsénico ocurre en el rango de $\mathrm{pH}$ de 6.5-8.5.

Tabla 4 Concentraciones de arsénico inorgánico determinadas en el PRM-3 a diferentes $\tau_{\text {FMADT }}$.

\begin{tabular}{cccc}
\hline $\begin{array}{l}\text { Lote procesado } \\
\text { por el FMADT }\end{array}$ & $\begin{array}{c}\text { Código } \\
\text { de la } \\
\text { muestra }\end{array}$ & $\tau_{\mathrm{FMADT}}$ & $\begin{array}{c}\text { Concentración de arsénico } \\
\text { en el PRM-3 en } \mu \mathrm{g} / \mathrm{L} \text { As }\end{array}$ \\
\hline Lote 2 (día 87) & As-1 & 8.41 & 0.00 \\
\hline Lote 2 (día 90) & As-2 & 9.76 & 0.00 \\
\hline Lote 1 (día 94) & As-3 & 14.00 & 0.00 \\
\hline Lote 2 (día 95) & As-4 & 8.23 & 0.00 \\
\hline Lote 1 (día 98) & As-5 & 13.68 & 0.00 \\
\hline Lote 2 (día 99) & As-6 & 10.23 & 0.00 \\
\hline Lote 2 (día 101) & As-7 & 6.57 & 0.00 \\
\hline Lote 2 (día 103) & As-8 & 7.70 & 0.00 \\
\hline Lote 1 (día 104) & As-9 & 16.34 & 0.00 \\
\hline Lote 2 (día 105) & As-10 & 7.80 & 0.00 \\
\hline Lote 1 (día 106) & As-11 & 16.32 & 0.00 \\
\hline Lote 1 (día 107) & As-12 & 12.85 & 0.00 \\
\hline Lote 2 (día 107) & As-13 & 8.17 & 0.00 \\
\hline Lote 1 (día 108) & As-14 & 40.67 & 0.00 \\
\hline Lote 2 (día 108) & As-15 & 6.71 & 0.00 \\
\hline
\end{tabular}

\section{Conclusiones}

Se requirieron de 31 días para que la capa biológica producida en el FBA alcanzara un apto desarrollo, lo cual fue evidente por la estabilización de la turbiedad en el efluente (PRM-2) del FBA. Durante el monitoreo del ST, se obtuvo que éste es eficiente en la remoción de la turbiedad al cumplir con las normas CAPRE, atribuido al desempeño del FBA, el cual presentó estabilidad al remover dicho parámetro. La disminución del $\mathrm{pH}$ en el FBA permite que del ST se obtenga agua que cumpla con el rango de $\mathrm{pH}$ establecido por CAPRE.

En base a que el manganeso fue el único parámetro que no cumplió con la NTON 05007 98 y considerando que la cantidad en que se excede no es muy significativa, se establece que el AC-3 es adecuada para ser acondicionada por medio de la filtración y después ser destinada al uso doméstico. Solamente la conductividad, dureza magnésica y magnesio no cumplieron con la normas CAPRE, pero a las magnitudes que estos parámetros excedieron su respectivo valor regulatorio las referencias o estudios no sugieren posibles efectos adversos a la salud humana, por lo tanto el ST suministra agua filtrada de buena calidad y que es apta para un proceso de consumo humano. El ST demostró las más altas eficiencias en la remoción de turbiedad, pero también removió eficientemente manganeso, amonio y sobre todo el arsénico. Desde otra perspectiva, se comprobó que el FBA puede remover turbiedad hasta en un $98 \%$. Además, como un nuevo hallazgo se determinó que el FBA remueve el amonio hasta en $62 \%$. Por su parte, el FMADT remueve arsénico inorgánico a concentraciones menores al valor máximo admisible establecido por CAPRE (10 $\mu \mathrm{g} / \mathrm{L} \quad \mathrm{As})$, aunque también remueve apreciablemente manganeso, nitrato y nitrato. 
Asimismo, un tiempo de residencia en el FMADT entre 6 y $7 \mathrm{~h}$ fue necesario para la remoción de arsénico, hasta una concentración menor que $10 \mu \mathrm{g} / \mathrm{L}$.

\section{Agradecimientos}

Los autores agradecen a la Organización Aqua Clara Nicaragua, ONG "El Porvenir", Ministerio de Salud (MINSA), Empresa Nicaragüense de Acueductos y Alcantarillados (ENACAL) y a la Universidad Nacional de Ingeniería (UNI) por la colaboración para la realización de este trabajo. También a la Lic. Aura Pérez Hernández por la asistencia con los análisis microbiológicos. Asimismo, se agradece por la asesoría, consultas, ayuda técnica y contactos con otras instituciones brindadas por Dr. Rafael Gamero, Dra. Indiana García, Mag. Elda Escobar, Ing. Juan Santos, y Lic. Alejandro Hernández.

\section{Referencias}

Arboleda, J. (2000). Teoría y práctica de la purificación del agua. Tomo I. Santa Fe de Bogotá, Colombia: McGraw-Hill.

Aurazo, M. (2004). Manual para el análisis básico de la calidad del agua de bebida. Lima, Perú: CEPIS/OPS/OMS/PUB/02.93.

Bang, S., Patel, M., Lippincott, L., Meng, X. (2005). Removal of arsenic from groundwater by granular titanium adsorbent. Chemosphere, Vol. 60, No. 3, pp. 389-397.

Buzunis, B.J. (1995). Intermittently operated slow sand filtration: a new water treatment Process. MSc Thesis: University of Calgary, Canada.

Carbotecnia (2010). ¿Qué es el carbón activado? Jalisco, México: Carbotecnia-Carbón Activado.

Centre for Affordable Water and Sanitation Technology, CAWST (2012). Biosand filter construction manual. Canada: Calgary.

Chapman, D. (1996). Water quality assessments: a guide to use of biota, sediments and water in environmental monitoring ( $2^{\text {nd }}$ Ed.). Great Britain: WHO, UNESCO/UNEP.

Giraldo, B. (2004). Guía de promoción y desarrollo comunitario para asegurar la calidad del agua en los países en desarrollo. Lima, Perú: OPS/OMS/CEPIS/PUB 04.104).

Guan, X., Du, J., Meng, X., Sun, Y., Sun, B., $\mathrm{Hu}, \mathrm{Q}$. (2012). Application of titanium dioxide in arsenic removal from water: A review. Journal of Hazardous Materials, Vol. 215-216, pp. 1-16.

Hach Company (2005). DR5000 Spectrophotometer: Procedures manual $\left(2^{\text {nd }}\right.$ Ed.). Germany: Hach@ company world.

Ngai, T., Murcott, S., Shrestha, R. (2004). Kanchan arsenic filter (KAF): research and implementation of an appropriate drinking water solution for rural Nepal.

OPS/OMS (2001). Series de informes técnicos número 8: Desigualdades en el acceso, uso y gasto con el agua en América Latina y el Caribe. Washington D.C.: OPS/OMS.

Rice, E.W., Baird, R.B., Eaton, A.D., Clesceri, L.S. (2005). Standard Methods for the Examination of Water and Wastewater $\left(21^{\text {st }}\right.$ Ed.). Washington D.C., USA: AWWA.

Rojas, R. (2002). Guía para vigilancia y control de la calidad del agua para consumo humano. Lima, Perú: OPS/CEPIS/OMS/PUB/02.79.

Valencia, E. (2007). Proceso de remoción de Arsénico (V) en soluciones acuosas utilizando $\mathrm{TiO}_{2}$, como material sorbente. México: Universidad Michoacana de San Nicolás de Hidalgo.

Wagtech (2012). Arsenator®: Digital Arsenic Test Kit. United Kingdom: Wagtech Water Technology Division.

Weiner, E. R. (2008). Applications of environmental aquatic chemistry: a practical guide (2nd Ed.). United States of America: CRC Press. 